\title{
Percutaneous visceral pleural biopsy with fenestrated cup biopsy forceps
}

\author{
P P Prabhudesai, A A Mahashur, S N Murudkar, R Ajay
}

Department of Chest

Medicine and

Environmental

Pollution Research

Centre,

Cardiovascular and

Thoracic Centre, King

Edward VII Memorial

Hospital, Bombay 400

012, India

P P Prabhudesai

A A Mahashur

S N Murudkar

R Ajay

Correspondence to:
Dr A A Mahashur (reprints will not be available)

Accepted 23 June 1992

\begin{abstract}
When there is an exudative pleural effusion often both the parietal and the visceral pleura are affected, but the usual practice is to perform a percutaneous parietal pleural biopsy alone for diagnosis. Percutaneous visceral pleural biopsy was carried out in 20 patients with exudative pleural effusions with fenestrated cup biopsy forceps. In all 20 biopsies pleural tissue was obtained and it was diagnostic in 19 cases. The procedure is painless and appears safe.
\end{abstract}

\section{(Thorax 1992;47:753-754)}

Pleural biopsy is a useful technique for the diagnosis of pleural effusions. Closed (percutaneous) pleural biopsy is a routine procedure, Copes or Abrams biopsy needle or the Vim-Silvermann, Tru-cut, or Raja needle being used..$^{1-3}$ All these biopsy needles, however, are designed to obtain samples of parietal pleura. The aim of this pilot study was to determine whether visceral pleural biopsy samples taken with cup forceps were associated with a high diagnostic yield and an acceptable complication rate.

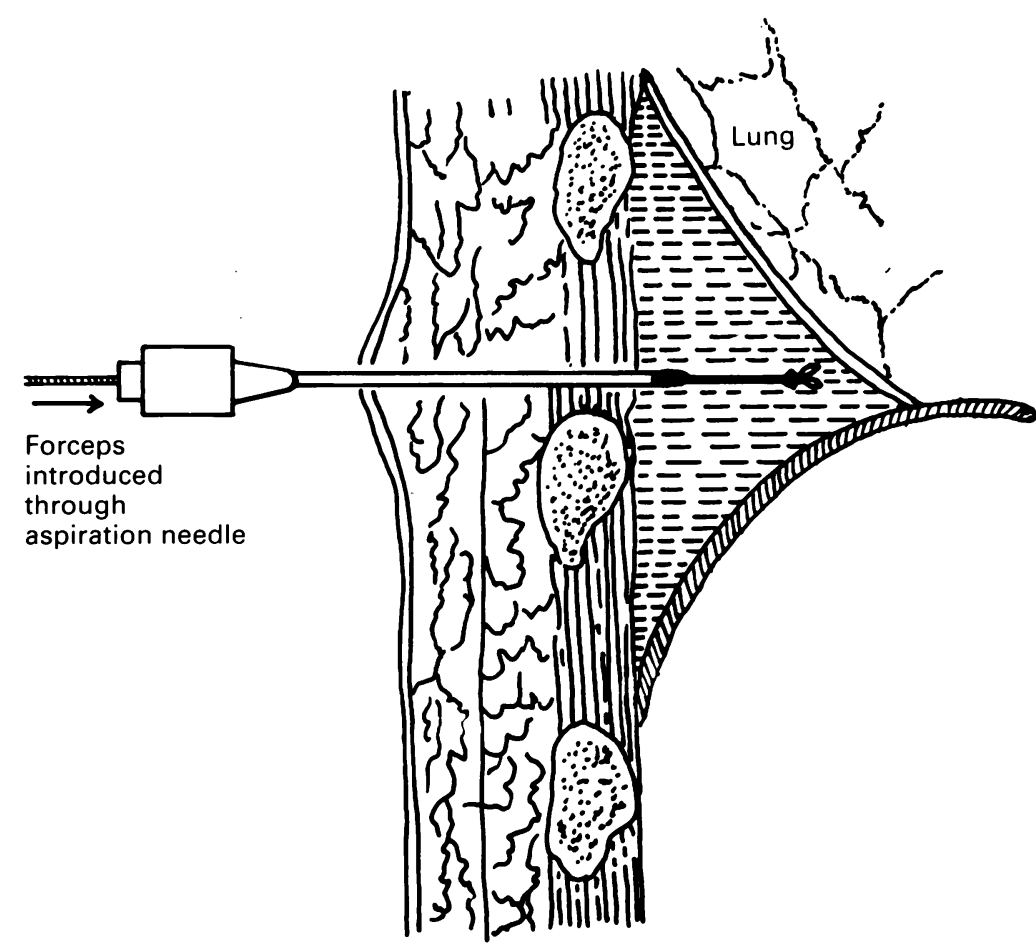

\section{Methods}

Twenty patients with pleural effusion were included in this study. The minimum size of effusion was one that outlined the costophrenic angle on a posteroanterior chest radiograph, with loss of convexity of the diaphragmatic outline. The skin at the biopsy site was cleaned and anaesthetised with $4-5 \mathrm{ml}$ of $2 \%$ lignocaine. A 15 gauge needle was introduced into the pleural cavity and its position confirmed by withdrawing fluid. A fenestrated cup biopsy forceps-FB-19C (Olympus)with an external diameter of $2 \mathrm{~mm}$ was introduced through the needle (figure). The foreceps was advanced until the lung was felt touching and receding from its tip during quiet respiration. The patient was then asked to hold his or her breath at the end of a normal inspiration. The forceps was advanced with the cup open and after resistance was felt as it touched the underlying lung a biopsy specimen was obtained, light pressure being used. Care was taken to avoid penetrating the lung parenchyma. The forceps was withdrawn and the mouth of the needle occluded with a finger to prevent air leaking into the pleural cavity. Samples were placed in $10 \%$ formalin. The forceps was immediately reintroduced into the pleural cavity for further biopsy samples, and this was done three or four times at different sites by changing the angle of the outer needle at the skin. The forceps was removed, leaving the aspiration needle inside the pleural cavity. Aspiration of pleural fluid was then continued and any haemorrhage noted. A chest radiograph was taken four hours after the procedure. The visceral pleural biopsy was repeated at a different site with the same technique if the initial report was not diagnostic.

\section{Results}

We studied 20 patients ( 18 male) with a mean age of $29 \cdot 3$ (range 15-43) years. In 16 patients the effusion was moderate to large, with an upper level above the anterior end of the third rib. Smaller effusions were seen in two patients, and in the remaining two the effusion was encysted. Six patients had additional lesions, either on the same side or on the opposite side from the effusion, in the form of infiltration, cavitation, or hilar lymphadenopathy. All the pleural effusions were exudative (protein more than $30 \mathrm{~g} / \mathrm{l}$ ) with lymphocytic predominance. Blood tinged effusions were seen in three patients; the remaining 17 were straw coloured. 
All patients easily tolerated the procedure. In three patients the biopsy was performed twice, and in one patient three times. The tissue obtained was about four to six times larger than that expected with a Copes needle. In three patients a small amount of lung tissue was taken along with pleural tissue. No major complications occurred after any biopsy. A small pneumothorax was seen in five of the 20 patients, which disappeared after closed needle aspiration. None of these patients needed intercostal tube drainage, or had lung tissue in the biopsy sample.

The diagnosis was tuberculosis in 15 patients and adenocarcinoma in three, squamous cell carcinoma in one, and non-specific pleuritis in one. Among the 15 patients with tuberculosis, the diagnosis was made after a second attempt at biopsy in two cases and after a third attempt in one.

\section{Discussion}

In malignancy and tuberculosis the visceral pleura is commonly affected-in malignancy more often than the parietal pleura. ${ }^{45}$ In studies of parietal pleural biopsy the proportion of adequate samples has ranged from $73 \%$ to $100 \% .^{1}$ This variation can be attributed to experience, technique, and the type of needle used. We have found no report on blind visceral pleural biopsy. The advantage of visceral pleural biopsy appears to be a high diagnostic yield, due to the fact that large samples are taken from different sites-which are, naturally, close to the underlying, presumably diseased, lung. Canto et al, ${ }^{6}$ in their study of thoracoscopy in pleural effusion, found various forms of inflammatory lesions in both parietal and visceral pleura-for example, acute hyperaemic pleura, subacute white or yellow plaques, fibrin deposits, and white-grey nodules. Biopsy of the visceral pleura in inflammatory and malignant lesions was found to be safe, with no increase in complication rate. ${ }^{6}$

Blind visceral pleural biopsy by our technique is a painless procedure and the cooperation obtained from the patients was good.

The procedure is simple and is quickly learnt. It appears safe as we did not encounter any important complication in the 20 patients undergoing biopsy. The diagnostic yield was high (19/20). A controlled study comparing this technique with parietal pleural biopsy is now required. Thoracoscopic pleural biopsy would undoubtedly be safer and yield more information. It is, however, more complex and requires more expertise, is more expensive, and is not widely practised as an initial procedure for diagnosing exudative effusions.

In conclusion, this pilot study suggests that visceral pleural biopsy with fenestrated cup biopsy forceps is a simple and safe method of obtaining the diagnosis in cases of exudative pleural effusion. Experience is so far too limited, however, to justify its widespread use in different populations of patients

We thank Dr A P Rao, superintendent of the group of tuberculosis hospitals, Sewree, Bombay, for giving us permission to carry out this project in his hospital. We also thank $\mathrm{Dr}$ P M Pai, dean of King Edward VII Memorial Hospital, for allowing us to publish this study.

1 Von Hoff DO, LiVolsi V. Diagnostic reliability of needle biopsy of the parietal pleura. Am J Clin Pathol 1975;64: biopsy

2 Berger HW, Mejia E. Tuberculous pleurisy. Chest 1973;

3 Raja OG, Agrawal V, Aldrich TK. Raja pleural biopsy needle, a comparison with Abram's needle in experimenta pleural effusion. Am Rev Respir Dis 1989;139:984-7.

4 Meyer PC. Metastatic carcinoma of the pleura. Thorax 1966; 21:437-43.

5 Stead W, Eichenholz A, Strauss HK. Operative and pathoogic findings in twenty four patients with syndrome of diopathic pleurisy with effusion, presumably tuberculous. Am Rev Respir Dis 1955;71:473-502.

6 Canto A, Blasco E, Casillas M, Zarza AG, Padilla J, Pastor J et al. Thoracoscopy in diagnosis of pleural effusion. Thorax 1977;32:550-4. 